\title{
Etat des Finances Publiques au Burkina Faso
}

\author{
Djibrihina Ouedraogo*
}

«Plus rien ne sera comme avant. Allons seulement». Tels sont les slogans qui ont sanctionné la chute du régime Compaoré en octobre 2014 à la suite de ce qu'on a appelé « l'insurrection populaire des 30 et 31 octobre $2014 »$. Ces slogans venaient symboliser la volonté du peuple burkinabè à mettre fin aux pratiques contraires à la démocratie et à la bonne gouvernance des finances publiques. Cela se manifesta dès la formation du premier gouvernement de la Transition politique qui avait pour mission d'assurer le retour à l'ordre constitutionnel normal. En effet, M. Adama Sagnon, proposé à la tête du département de la culture et du tourisme, sera contraint à démissionner à la suite de manifestations populaires. Il lui a été notamment reproché un manque d'éthique et du sens de la justice, en raison du fait qu'il a déclaré un non-lieu sur l'affaire Norbert Zongo (journaliste d'investigation assassiné le 13 décembre 1998) alors qu'il était procureur du Faso. Cette exigence de la gouvernance vertueuse a donné lieu à un renforcement du rôle des structures de contrôle. C'est ainsi que l'ASCE-LC s'est notamment constitué comme la vigie (gendarme) des finances publiques par la dénonciation des pratiques de mauvaise gestion au sein des administrations publiques et que l'Assemblée nationale n'a pas cessé depuis lors de multiplier les commissions d'enquête parlementaire sur des thématiques diverses qui ont eu des échos retentissants.

Au regard de ces évolutions politiques et sociales récentes, il apparait intéressant de marquer un temps d'arrêt pour évaluer la performance des finances publiques burkinabè. Car, comme le disait Jean Bodin dans les six livres de la Républiques « l'argent est le nerf de l'État ». Concernant la situation des finances publiques, il convient de noter que d'une manière générale, elle présente des caractéristiques assez identiques à celles des autres pays de la sous-région ouest africaine, même s'il est bien entendu qu'il existe des spécificités. C'est ainsi que depuis cinq ans le pays connaît un taux de croissance moyen du PIB de 6\%; la dette publique est plus ou moins bien maitrisée avec un ratio dette publique rapporté au PIB inférieur à 40\% donc inférieur à la norme communautaire de $70 \%$. Le budget 2019 présente un déficit qui répond à la fois aux exigences des partenaires techniques et financiers et à la norme UEMOA d'un ratio solde budgétaire rapporté au PIB inférieur à $3 \%$.

Cependant derrière ces chiffres qui paraissent bien flatteurs, il existe bien une triste réalité, le Burkina Faso reste l'un des pays les plus pauvres du monde. Au classement de l'indice de développement humain de 2018, il occupe la 183ème place sur 189 pays classés et quatre Burkinabè sur dix (4/10) vivent encore en dessous du seuil de pauvreté estimé à 153000 F.CFA (environ 278 dollars US) par an et par individu.

Cette situation peu reluisante s'explique par le fait que la maîtrise des finances publiques reste assez délicate. Dans ce sens d'ailleurs, dans sa déclaration de politique géné-

* Enseignant-chercheur, Maître-assistant, Université Ouaga 2. 
rale, prononcée le 18 février 2019, le nouveau Premier ministre, Christophe Dabiré, expliquait les causes profondes de cette situation dans «la faible productivité des secteurs de production » qui commande qu' « un accent particulier soit mis sur l'amélioration de la mobilisation des recettes publiques et sur la maitrise de l'évolution des dépenses publiques ».

Le constat opéré par le Premier ministre laisse ainsi percevoir les deux tendances majeures actuelles des finances publiques burkinabè. Ces dernières sont caractérisées par une gestion dispendieuse des recettes publiques (II) alors qu'elles connaissent une faible mobilisation (I).

\section{I- Une faible mobilisation des ressources publiques}

A l'image de la plupart des pays membres de l'UEMOA, le niveau de mobilisation des recettes fiscales et budgétaires ordinaires présente un niveau relativement faible. Deux causes principales peuvent être soulignées : un système fiscal limité (A) se trouve éprouvé par une résistance fiscale caractérisée (B).

\section{A- Un système fiscal limité}

Le système fiscal burkinabè apparait limité à plusieurs égards.

D'abord, il est marqué par un faible taux de pression fiscale. En 2018, le taux de pression fiscale est estimé à $17,8 \%$, ce qui est largement en dessous de la norme communautaire UEMOA qui est de $20 \%$. Avec la loi de finances pour 2019 qui a notamment élargi la base d'imposition avec la création de nouveaux impôts et le relèvement de certaines taxes existant, le taux de pression fiscale devrait se situer autour de la norme communautaire, soit $20,22 \%$. Il est prévu à 20,92 en 2020 et $21,36 \%$ en 2021 . En valeur absolue, les recettes recouvrées s'élevait à seulement 594 milliards ( 1 milliard 100 millions) en 2016 contre 673 milliards (1 milliard 200 millions) en 2017. En 2018, les prévisions étaient de 749 milliards (1 milliard 400 millions de dollars) Autrement dit, le système fiscal n'arrive pas à imposer de façon optimale l'ensemble des activités économiques. Cela tient notamment à la difficulté d'imposer le secteur informel mais également aussi à l'importance des niches fiscales. Dans ce dernier cas, le code des impôts prévoit de nombreuses dérogations ou faveurs fiscales, notamment avec la politique des dépenses fiscales.

Ensuite, les techniques de recouvrement de l'impôt apparaissent peu performantes. Cela tient notamment à la structure encore relativement dynamique de l'Administration fiscale. A cet égard, il existe une forte centralisation de l'Administration fiscale dont le personnel est composé d'un nombre limité d'agents publics. Encore qu'ils sont mal repartis : l'essentiel des 2300 agents des impôts est constitué d'agent de rang A, donc plus d'inspecteurs que de contrôleurs. Aussi, l'Administration fiscale ne dispose pas de moyens pour pouvoir comptabiliser toutes les personnes imposables avec le niveau réel du revenu de chacun. Il convient de noter le développement de moyens modernes de paiement des impôts avec les 
télé déclarations, les paiements par mobil money et la mise en place à venir de logiciel qui devrait permettre aux banques de connaitre la situation fiscale de leurs clients afin d'effectuer des prélèvements à la source au profit du Trésor. En tous les cas, l'informatisation et l'interconnexion entre les grandes administrations fiscales, c'est-à-dire douane et fisc, est quasi inexistante, ce qui favorise la fraude fiscale.

L'efficacité des recouvrements apparaît encore délicate en raison de l'importance de la corruption au sein des administrations fiscales.

Enfin, les difficultés du recouvrement posent la question du mode de recouvrement. En effet, lorsque le recouvrement ne se fait pas à la source, les contribuables montrent très peu d'enthousiasme à honorer la dette fiscale. Tel est l'exemple topique de la taxe de résidence qui n'est acquitté en général que lorsque l'on veut se présenter devant le maire avec son partenaire. En réaction à cette situation, l'Administration fiscale a procédé d'office au prélèvement à la source de la taxe de résidence. Outre son caractère illégal, la démarche met en évidence la question de l'égalité devant l'impôt et devant les charges fiscales dès lors qu'il n'existe aucun moyen de prélever à la source les opérateurs économiques qui évoluent à leurs propres comptes.

Ce refus d'acquitter la taxe de résidence pose de façon plus générale la problématique de la résistance fiscale.

\section{B- Une résistance fiscale caractérisée}

La faiblesse des recettes publiques s'explique par ailleurs par l'importance de la résistance fiscale. Celle-ci est due à une faible adhésion psychologique et sociologique à l'obligation fiscale. A cet égard, on a l'impression que personne ne veut payer les impôts. Les statistiques montrent dans ce sens que l'effort fiscal est supporté par la minorité des contribuables (30\% des contribuables paient environ 95\% des recettes fiscales). Comme le faisait remarquer un éminent professeur burkinabè, ancien DG des impôts : "la psychologie du contribuable burkinabè est marqué par la croyance en la divine providence de l'État». Ce qui explique que «chacun consomme tout ce qu'il gagne et attend tout de Dieu, de l'État ou des parents et amis ».

Cette résistance fiscale peut prendre plusieurs formes.

D'abord, on constate un ras-le-bol généralisé qui se manifeste par une contestation grandissante de l'impôt sur les revenus salariaux. Ainsi, depuis 2017, les salariés du privé et les syndicats des fonctionnaires demande la suppression de l'impôt sur le revenu. Le paradoxe tient ici au fait qu'il exige une augmentation des salaires. En 2018, les députés semblaient vouloir revenir sur leur décision adoptée en 2016 en vue de prélever l'impôt sur le revenu sur leurs indemnités parlementaires.

Ensuite, la résistance fiscale a une autre figure dans la fraude fiscale qui conduit un grand nombre de contribuables à occulter leurs activité ou le montant exact de leur revenu afin d'échapper ou de diminuer le montant de l'impôt normalement dû. Cette fraude fiscale peut elle-même se présenter sous diverses formes. Le rapport de la Commission d'enquête 
parlementaire sur la fraude fiscale effectuée en 2015 par le Conseil national de la Transition (CNT) a révélé par exemple des fraudes en douanes qui se manifestent par le fait que certains opérateurs économiques acheminent des marchandises avec des motos qui échappent aux postes frontières grâce à des pistes et chemins qu'ils suivent à l'intérieur de la forêt. Dans le même sens, il faut souligner également la multiplication de sociétés taxis. Ce sont des montages fiscaux réalisés avec la complicité malveillante de certains agents du fisc en vue d'empêcher le reversement de la TVA normalement due.

Dans la dynamique des société-taxis, il convient de noter que le secteur informel constitue une sorte de lit de la fraude fiscale. En effet, de nombreux opérateurs y trouvent volontairement refuge afin de réduire le montant des impôts dus.

Enfin, la résistance à l'impôt se manifeste par les révoltes fiscales (manifestation des « chemises rouges » en novembre 2018 contre la hausse des taxes sur les produits pétroliers) et par le développement des passe-droits fiscaux au sein de l'Administration fiscale et douanière. En effet, le simple fait d'avoir un proche dans ses administrations peut être mis à profit pour échapper au paiement de l'impôt.

\section{II- Une gestion dispendieuse des ressources publiques}

Cette gestion dispendieuse des finances publiques peut être remarquée à un double point de vue. Non seulement, il y a une mauvaise utilisation des ressources publiques (A) mais encore, il persiste des détournements de deniers publics (B).

\section{A- Une utilisation inconséquente des ressources publiques}

La mauvaise utilisation des ressources publiques est surtout symbolisée ces dernières années par la difficulté à maîtriser la masse salariale qui a atteint des chiffres record éloignés de la norme communautaire UEMOA. Alors que la norme communautaire exige un ratio masse salarial rapporté aux ressources propres de $-35 \%$, ce ratio est fixé à $46,64 \%$ en 2019 , à 46,09\% en 2020 et à 45 en 2021. En valeur absolue, la masse salariale s'élevait à 700 milliards de FCFA (1 milliards 375 millions de dollars) contre 835 milliards FCFA (1 milliard 500 millions de dollars) prévus pour 2019, soit une hausse de 19,1\%. Cette masse salariale représente à elle seule $37,7 \%$ des dépenses budgétaires contre $25,5 \%$ en 2018 . En comparaison les dépenses d'investissement s'élève à seulement 628 milliards de FCFA (1 milliard 140 millions de dollars), soit $28,38 \%$ contre $44,44 \%$ en 2018 . Autant dire que l'investissement est éprouvé par la hausse de la masse salariale. Ce qui est inquiétant pour un pays comme le Burkina Faso qui est caractérisé par un manque d'infrastructures et de services publics. Contrairement à la logique de la fongibilité asymétrique imposée par l'UEMOA, il apparaît que ce sont les crédits initialement destinés à la réalisation d'opérations d'investissement qui viennent abonder les crédits relatifs aux dépenses de personnel. Le comble, c'est qu'il est souvent fait recours à des emprunts sur le marché financier régional 
et international pour faire face à ces dépenses de personnel, ce qui induit un mauvais usage de l'emprunt public.

Plusieurs raisons expliquent cette explosion de la masse salariale.

D'abord, en raison de l'usure du régime Compaoré qui a duré 27 ans (plus d'un quart de siècle), les crises dans la fonction publique se soldaient par l'octroi de statuts particuliers à plusieurs corps de fonctionnaires. En réalité, l'objet principal de ces statuts particuliers était la revalorisation des salaires. Le nouveau statut général de la fonction publique, adopté sous la Transition politique, n'a pas mis fin à l'hémorragie. De fait, ce statut général reniait sa vocation générale dès l'origine puisqu'il excluait de son champ d'application pas moins de sept catégories de fonctionnaires.

Le régime démocratiquement élu à la suite de la Transition s'est enlisé dans cette dynamique avant de se rendre compte en juin 2018 de l'indispensable maîtrise de la massa salariale. Deux corps de fonctionnaires ont symbolisé cette situation. Celui des magistrats dont les salaires sont passés du simple au double dès mars 2016. Puis, celui des agents du ministère des finances qui, en plus de leur salaire, bénéficient chaque année d'une prime issue de la répartition d'une cagnotte commune appelée fonds commun. $\mathrm{Si}$, ces fonds communs devaient à l'origine servir à motiver les agents chargés du recouvrement des fonds publics, la pratique a donné lieu à des dérives graves quant à la maitrise des finances publiques. C'est ainsi qu'initialement annexé aux pénalités dues par les contribuables, ces fonds communs ont été ensuite adossés à des ressources budgétaires. Leur montant va s'accroître de sorte à concurrencer sinon dépasser le salaire annuel de ces fonctionnaires.

En mars 2018, devant les députés, la ministre des finances devait faire remarquer que l'évolution des fonds communs donne du tournis : le FC est passé de 20,11 milliards de FCFA (37 millions de dollars) en 2012 à 55 milliards de FCFA (100 millions de dollars) en 2017; en 2016, la masse salariale de l'ensemble des agents du ministère des Finances était de 23,851 milliards de FCFA (43 millions de dollars) contre un Fonds commun de 26, 967 milliards (48 millions de dollars); on perçoit donc que le fonds commun (FC) est nettement au-dessus de la masse salariale.

Au-delà de la mauvaise maîtrise de la masse salariale, il faut également relever certaines habitudes qui ne conduisent pas à une utilisation rationnelle des ressources publiques. Ainsi, au niveau de l'exécutif, certains ministères et institutions n'avaient pratiquement d'objet de fonctionnement. Ça été le cas avec la création d'un ministère d'État chargé des affaires présidentielles et de l'institution du Haut représentant du chef de l'Etat, institution extraconstitutionnelle créée par voie de décret présidentiel. Heureusement, ce ministère et ce Haut représentant disparaisse avec le dernier Gouvernement installé en janvier 2019.

Dans cette dernière dynamique, on peut souligner aussi le syndrome des ateliers et séminaires. Ces ateliers et séminaires de travail, étant en général assortis de paiement de frais de mission lorsqu'ils ont lieu en dehors du service bénéficiaire, il s'en suit que les agents publics ne sont plus disposés à travailler en dehors du cadre de ces ateliers et séminaires. Or, lors de ces séminaires, il est automatiquement fait recours à un restaurateur (pause-café) qui est rémunéré sur les fonds publics. Quel gaspillage! 
Enfin, pour leur part, non seulement les députés rétrocèdent difficilement les prêts consentis par l'Etat mais encore s'octroie des primes et des avantages considérables, telle que les primes de Noël qui s'élève à un montant de 3 millions par députés pour une Assemblée qui en comporte 127, soit 127x3 millions=381 millions FCFA (693 milles dollars).

En tout état de cause, devant l'explosion des dépenses face à la rareté des ressources publiques, le Gouvernement a mis en place une Conférence nationale sur la réforme du système de rémunération des agents publics et autres avantages pécuniaires services. Cette conférence qui regroupait toutes les forces vives de la Nation a adopté des recommandations fortes dont les plus emblématiques sont pris en comptes dans le budget 2019. Il en est ainsi du montant des fonds communs qui sont annexés aux pénalités sans qu'ils ne puissent dépasser $25 \%$ de la masse salariale des fonctionnaires qui en bénéficient.

A cette mauvaise répartition de ces maigres ressources publiques, il faut rajouter l'effet nocif des détournements de deniers publics.

\section{B- Une persistance des détournements des deniers publics}

Concernant le détournement des deniers publics, un contraste saisissant est à noter. En effet, si la Transition politique a renforcé et dynamisé le rôle des structures de contrôle, on note une persistance des pratiques illégales de gestions des fonds publics. Pire, le rapport 2017 de l'ASCE montre une détérioration des indicateurs auxquels il faut ajouter les pratiques de mauvaise gestion constatées au niveau de certaines structures chargées de garantir une bonne gestion des finances publiques, à savoir l'Assemblée nationale et la Cour des comptes.

Concernant les pratiques de mauvaise gestion financière, dans son rapport de mars 2018 portant sur l'année 2017, l'ASCE fait le constat d'une amplitude des dépenses irrégulières relatives à la commande publique et à la consommation du carburant dans les services publics. Pour la commande publique, l'ASCE fait le constat d'une augmentation du pourcentage des commandes passées par entente directe contraire à la norme communautaire UEMOA. Alors que la norme communautaire fixe à un maximum de $15 \%$ le pourcentage des commandes passées par entente directe, ce taux était de 35,59\% en 2017 contre 15,46\%, d'où une forte dégradation de cet indicateur de 20,13\%. Pour les seuls marchés relatifs au carburant, les marchés passés par entente directe représentait une proportion de $2,2 \%$ en 2016 contre $3,11 \%$ en 2017 , soit une dégradation de $0,91 \%$. L'ASCE note en toute logique la dégradation des indicateurs d'une année sur l'autre corrobore la perception d'une augmentation des pratiques de corruption au Burkina Faso entre 2016 et 2017.

Cette situation est d'autant plus préoccupante que des cas de mauvaise gestion et de malversation financière ont été découverts à l'Assemblée nationale et à la Cour des comptes. Concernant l'Assemblée nationale, Au cours de l'année 2017, les députés (tout bord politique confondu) et le personnel de l'Assemblée nationale ont été épinglés pour près de 235 missions effectuées hors du pays sans en fournir les pièces justificatives. Suite à la dénonciation par la presse, une commission ad-hoc d'audit instituée par l'Assemblée na- 
tionale a noté l'absence d'un plan de passation de marchés adopté par une commission et d'un manuel de procédures budgétaires, financières et comptables.

Concernant la Cour des comptes, en 2017, un audit réalisé par un cabinet privé a révélé des dépenses inexpliquées d'environ 56 millions de francs CFA (102 milles dollars). La Cour des comptes n'a pas produit les pièces justificatives de cette importante somme.

Ces exemples peuvent être multipliés. Leur persistance s'explique par le fait que auteurs de ces pratiques ne sont pas efficacement sanctionnés du fait de la complaisance des autorités politiques ou du fait des dysfonctionnements de la justice qui n'est pas à l'abri de la corruption. C'est ainsi qu'en 2012 dans le cadre de l'affaire, l'ex-DG de la douane, coupable d'avoir entreposé au domicile de son neveu des cantines comportant d'importantes sommes d'argent et d'objets de valeurs estimés à quelque 2 milliards de FCFA (4 millions de dollars) a été relaxé et même obtenu un mandat municipal dans son village.

Toutefois, même s'il persiste des cas de mauvaises gestions, il est juste de dire que ces malversations sont de moindre ampleur que par le passé.

\section{Conclusion :}

Il y a un contraste saisissant entre la rareté des ressources et leur mauvaise utilisation. L'explosion de la masse salariale doit sonner comme une piqure de rappel aux pouvoirs publics pour tendre vers une gestion plus orthodoxe des finances publiques. A cet égard, il convient à nos autorités et à la population burkinabè de ne pas oublier cette sagesse transmise par nos ancêtres : "L'argent se cherche avec idiotie mais se dépense avec intelligence ». 